\title{
Variational Calculation of Low-energy Positron Life Time in Xe Atoms
}

\author{
Mahasen M. Abdel-Mageed* \\ Physics Department, Faculty of Science, Ain Shams University, Cairo, Egypt
}

(Received on 3 July, 2008)

\begin{abstract}
The least-squares variational method (LSVM) is used for determining trial wavefunctions representing lowenergy positron-xenon elastic scattering, which are used to determine the positron lifetime. The trial function is taken to depend on several adjustable parameters, and is improved iteratively by increasing the number of terms. The bound state wavefunctions are obtained using Hartree-Fock-Slater method. The $2 \gamma$-rays annihilation rates of the positron in xenon atoms below Positronuim (Ps) formation threshold are calculated. Polarization potential $V_{p l}(r)$ is applied to enhance annihilation rates and lifetime. The present results of annihilation parameters are consistent with semiempirical, theoretical and experimental results.
\end{abstract}

Keywords: Positron Annihilation; Bound wavefunction; Scattered wavefunction; Variational Method

\section{INTRODUCTION}

An important and interesting feature of low-energy positron collisions with atoms and molecules is the possibility of annihilation of the positron with one of the electrons in the target. This has been the subject of extensive experimental and theoretical work [1-2]. Only elastic scattering or direct annihilation are possible when the positron incident with energy less than the positronuim formation threshold.

Positron annihilation is a good tool to give useful information about matter. It is important in the study of metals [3], alloys [4], polymers [5] and super-conductors [6]. Particularly, it has been confirmed that positron annihilation lifetime technique is one of the most effective nondestructive tools for providing valuable information about the electronic structure and defects of materials and determination of the Fermi surface in the single crystal which is useful in band structure calculation $[7,8]$.

A unique aspect of these interactions is the emission of $\gamma$ rays when a positron annihilates with an electron. This signal provides information about the interaction, and it is the basis of many types of measurements. For example, this signal has been used to characterize defects and interfaces in solids $[9,10]$.

When positrons annihilate with atoms they annihilate predominantly with valence electrons because of the repulsive potential exerted on the positron by the nuclei. However, a small fraction of the positrons can tunnel through this repulsive potential and annihilate with inner-shell electrons. An important implication of the inner-shell annihilation is the emission of Auger electrons and consequent formation of doubly ionized atoms. The positron-induced Auger electron emission has been observed in condensed matter [11].

There have been several experimental and theoretical studies on positron-xenon interaction at low energy has been the subject of several experimental works. One-electron HartreeFock with random phase approximations [12] was employed to calculate the scattering cross section of positrons on noble gas and alkali atoms. The many-body theory methods [13] are applied to study the interaction of low-energy positrons with Xe atoms which described by means of a non-local energy dependent-correlation potential. The polarized or-

*Electronic address: mabdelmageed@hotmail.com bital method[14] is applied to calculate the elastic scattering cross section and annihilation rates of low energy positrons on xenon atoms below the positronuim formation threshold. The annihilation rate of room-temperature positrons in low-density gaseous xenon-nitrogen mixtures has been measured[15].The annihilation rates were measured for positron in xenon below Positronuim formation threshold [16].

The least squares variational method was applied to low energy positron scattering from $\mathrm{H}, \mathrm{He}$ [17] , $\mathrm{Ar}$ [18], and $\mathrm{Kr}$ [19]. It was applied to obtain the wave function of the continuum Auger electron emitted from an ionized Ne atom [20] and developed to study positron-H molecules scattering [21]. Good results were obtained in the former calculations.

In this work the least squares variational method have been applied to positron-xenon interaction at low energy below Positronuim Ps formation threshold. For xenon, the Ps formation threshold is at $5.33 \mathrm{eV}$ (momentum $\sim 0.63$ ao-1). This threshold is equal to the ionization energy of the atom minus the binding energy of a positronuim atom, namely half a Rydberg, $6.8 \mathrm{eV}$. The interaction potential between the positron and the atom is considered as a static potential and the polarization potential is taken into account. In this method the trial scattering function is taken to depend on several adjustable parameters, and is improved iteratively by increasing the number of terms. The scattering wavefunction is used to calculate the annihilation parameter $Z_{\text {eff }}$ at each orbit of the target as well as the positron lifetime.

\section{THEORY}

Annihilation into two $\gamma$-rays is far more probable than into three $\gamma$-rays. The annihilation rate in a gas is [22]

$$
\lambda=\pi r_{0}^{2} c \rho Z_{e f f}(k),
$$

and the positron life time is given by

$$
\tau=\frac{1}{\lambda}
$$

where $r_{o}=e^{m} 2 /\left(m c^{2}\right)$ is the classical radius of the electron, $c$ the speed of light, $\rho$ is the density of electrons per atom available to the positron for annihilation and $k$ is the positron momentum. $Z_{e f f}$ is the effective annihilation parameter. The value of $Z_{e f f}$ which varies with the momentum of 
the positron, is a measure of the probability of the positron being at the same position as one of the target electrons. It is calculated from the elastic scattering wavefunction for the positron - target system as follows:

$$
Z_{e f f}(k)=\left\langle\Psi\left(x, r_{N} ; k\right)\left|\sum_{i=1}^{N} \delta\left(r_{i}-x\right)\right| \Psi\left(x, r_{N} ; k\right)\right\rangle
$$

where $\Psi\left(x, r_{N} ; k\right)$ is the scattering wavefunction, including all partial waves, for the system made up of the incident positron with wave vector $k$ and the target atom. $x$ and $r_{N}$ stand for the position vectors of the positron and the target (composed of $\mathrm{N}$ electrons ), respectively. A good agreement between the calculated value of $Z_{e f f}$ and an accurate experimental value, derived from measurements of the annihilation rate, is therefore an important test of the quality of the scattering wavefunction.

\section{II.1. The least-Squares Variational Method and Trial Wavefunction}

For s-wave scattering process, the variational treatment [17],[23] starts by defining a trial wavefunction $\left|\Psi_{t}^{n}\left(x, r_{N} ; k\right)\right\rangle$. It consists of two multiplicative wavefunction

$$
\left.\left|\Psi_{t}^{n}\left(x, r_{N} ; k\right)\right\rangle=\left|\Phi_{T}\left(r_{N}\right\rangle\right| \Psi_{S c}^{n}(x ; k)\right\rangle
$$

where $\left|\Phi_{T}\left(r_{N}\right)\right\rangle$ represents the target in its ground-state and $\left|\Psi_{S c}^{n}(x ; k)\right\rangle$ is the positron scattering wavefunction which is composed of the angular part $\left(Y_{0,0}=\sqrt{1 / 4 \pi}\right)$ multiplied by the radial part $\left(\Psi_{P}^{n}(x ; k)\right)$. We have

$$
\left|\Psi_{P}^{n}(x ; k)\right\rangle=a^{n}|\widehat{S}(x ; k)\rangle+b^{n}|\hat{C}(x ; k)\rangle+\sum_{i=1}^{n} d_{i} \chi_{i}(x)
$$

$|\hat{S}(x ; k)\rangle$ and $|\hat{C}(x ; k)\rangle$ are the regular and irregular parts of the scattering wavefunction, respectively. $|\hat{C}(x ; k)\rangle$ should contain a cut-off function to avoid the singularity at the origin. This cut-off function will tend to zero at the origin and to unity at infinity. $\left|\chi_{i}(x)\right\rangle$ is a quadratic integrable wavefunction. $\left|\Psi_{P}^{n}(x ; k)\right\rangle$ has to satisfy the boundary conditions:

$$
\begin{gathered}
\left|\Psi_{P}^{n}(0)\right\rangle=|0\rangle \\
\left|\Psi_{P}^{n}(x ; k)\right\rangle \stackrel{x \rightarrow \infty}{\longrightarrow} a^{n}|\hat{S}(x ; k)\rangle+b^{n}|\hat{C}(x ; k)\rangle
\end{gathered}
$$

$a^{n} b^{n}$ and $d_{i}$ are variational parameters. In this case the reactance matrix $R_{11}$ contains a single element which is identical with the tangent of the s-wave scattering phase shift $\eta_{0}$ and is calculated by

$$
R_{11}=\tan \eta_{0}=b^{n} / a^{n}
$$

The s-wave elastic scattering trial wavefunction for the system may be written in abbreviated form as:

$$
\left|\Psi_{t}^{n}\right\rangle=|S\rangle+R_{11}|C\rangle+\left|\phi_{n}\right\rangle
$$

where $S$ is the regular part ;

$$
S=\hat{S} \cdot \Phi_{T}\left(r_{N}\right)=\frac{1}{\sqrt{4 \pi}} \operatorname{Sinc} \beta \cdot \Phi_{T}\left(r_{N}\right),
$$

Where, $\operatorname{Sin} c \beta=\frac{\operatorname{Sin} \beta}{\beta}, \beta=(k x)$. The function $\mathrm{C}$, consists of a cut-off function and the irregular part of the asymptotic solution. It has the form

$C=\hat{C} \cdot \Phi_{T}\left(r_{N}\right)=\frac{1}{\sqrt{4 \pi}}(1-\operatorname{Exp}(-\alpha x))(\operatorname{Cosc} \beta) \Phi_{T}\left(r_{N}\right)$,

where $\operatorname{Cosc} \beta=\frac{\operatorname{Cos} \beta}{\beta}$ and $\alpha$ is an adjustable (free ) parameter which is selected from the values that give a plateau of $R_{11}$ ( see ref. [17] P.73 ). $\Phi_{T}\left(r_{N}\right)$ is the target ground state wavefunction which can be expressed as a Slater determinant of mutually orthonormal one-electron wavefunction $u_{i}$ in the form:

$$
\Phi_{T}\left(r_{N}\right)=\frac{1}{\sqrt{N}} \operatorname{det}\left[u_{1}\left(r_{1}\right) u_{2}\left(r_{2}\right) u_{3}\left(r_{3}\right) \ldots . . u_{z}\left(r_{z}\right)\right]
$$

where $N$ is the total number of electrons. According to the central field model [24], $u_{i}\left(r_{i}\right)$ can be expressed as

$$
u_{i}\left(r_{i}\right)=\frac{1}{r_{i}} R_{n_{i} \ell_{i}} Y_{\ell_{i} m_{i}}\left(\hat{r}_{i}\right) \zeta(\sigma)
$$

where $R_{n_{i} \ell_{i}}$ is the radial wavefunction, which is the solution of the equation:

$$
\left[-\frac{d^{2}}{d r_{i}^{2}}+\frac{\ell_{i}\left(\ell_{i}+1\right)}{r_{i}^{2}}+V_{i}\left(r_{i}\right)\right] R_{n_{i} \ell_{i}}=\varepsilon_{i} R_{n_{i} \ell_{i}}
$$

where $V_{i}\left(r_{i}\right)$ is the assumed potential energy function for the field in which the atomic electron $i$ moves. These functions are generated from Cowan program using Hartree-FockSlater method [24] with Hartree plus statistical exchange approximated potential.

$Y_{\ell_{i} m_{i}}\left(\hat{r}_{i}\right)$ are the usual spherical harmonics and $\zeta(\sigma)$ stands for the spin wavefunction of the orbit $i$ such that $n_{i}, \ell_{i}$ and $m_{i}$ are the corresponding principal, orbital and magnetic quantum numbers. The Hilbert-space part $\left|\phi_{n}\left(x, r_{N}\right)\right\rangle$ possesses the form

$$
\left|\phi_{n}\left(x, r_{N}\right)\right\rangle=\Phi_{T}\left(r_{N}\right) \cdot \sum_{i=1}^{n} d_{i}\left|\chi_{i}(x)\right\rangle=\sum_{i=1}^{n} d_{i}\left|\phi_{i}\right\rangle
$$

where

$$
\chi_{i}=x^{i} \operatorname{Exp}(-\alpha x) \text { and } \phi_{i}=\chi_{i} \Phi_{T}
$$

$n$ refers to the dimension of the Hilbert-space part of the trial wavefunction The next step in the variational treatment is to select a proper test-wavefunction $\left|\phi_{S}\right\rangle$ and define the functional

$$
\left\langle\phi_{S}|H-E| \Psi_{t}^{n}\right\rangle=V
$$


The linear variational parameters $R_{11}$ and $d_{i}$ are chosen according to the following variational principle:

$$
\delta V^{2}=0
$$

Thus, they are chosen following to a least-squares variational principle in which all projections of the vector $(H-E)\left|\Psi_{t}^{n}\right\rangle$ on $\left|\phi_{S}\right\rangle$ are minimum. The test wavefunction $\left|\phi_{S}\right\rangle$ is constructed [25-26] by:

$$
\left|\phi_{S}\right\rangle=\left\{|S\rangle,|C\rangle,\left|\phi_{j}\right\rangle ; j=1,2, \ldots . . n\right\} .
$$

In this case we have the system of projections

$$
\begin{aligned}
& (S \mid S)+R_{11}(S \mid C)+\sum_{i=1}^{n} d_{i}\left(S \mid \phi_{i}\right)=V_{1} \\
& (C \mid S)+R_{11}(C \mid C)+\sum_{i=1}^{n} d_{i}\left(C \mid \phi_{i}\right)=V_{2} \\
& \left(\phi_{j} \mid S\right)+R_{11}\left(\phi_{j} \mid C\right)+\sum_{i=1}^{n} d_{i}\left(\phi_{j} \mid \phi_{i}\right) \\
& =V_{j+2} ; j=1,2, \ldots . . n
\end{aligned}
$$

and the LSVM implies :

$$
\delta \sum_{j=1}^{n+2} V_{j}^{2}=0
$$

The minimization of $\sum_{j=1}^{n+2} V_{j}^{2}=0$ guarantees that the vector $(H-E)\left|\Psi_{t}^{n}\right\rangle$ has a minimum length. The variational parameters are obtained by applying this variational principle. The matrix elements required for the employment of the LSVM, namely $(S \mid S),(S \mid C),\left(S \mid \phi_{i}\right),(C \mid S),(C \mid C)$, $\left(C \mid \phi_{i}\right),\left(\phi_{j} \mid S\right),\left(\phi_{j} \mid C\right)$, and $\left(\phi_{j} \mid \phi_{i}\right)$, have the general form [17]:

$$
\begin{aligned}
(g \mid f) & =\langle g|E-H| f\rangle=\langle g|\hat{H}| f\rangle=\langle g \mid \hat{f}\rangle \\
& =\int_{0}^{\pi} \operatorname{Sin} \theta d \theta \int_{0}^{2 \pi} d \varphi \int_{0}^{\infty} r^{2} g \hat{f} d r
\end{aligned}
$$

The operator $\hat{H}$ possesses the form

$$
\hat{H}=(E-H)
$$

The total Hamiltonian ( in Rydberg units ) of positron-target atom system has the form

$$
H=H_{T}-\nabla_{x}^{2}+V_{i n t}(r, x)+V_{p l}(r)
$$

where $H_{T}$ being the Hamiltonian for the target atom, $\nabla_{x}^{2}$ is the kinetic energy operator for the incident positron, and $V_{i n t}(r, x)$ stands for the static interaction potential between the positron and the target. The polarization potential $V_{p l}(r)$ is given the form [27]

$$
V_{p l}(r)=-\alpha_{d}\left(1-\exp \left(-r^{6} / r_{c}{ }^{6}\right) / 2 r^{4}\right.
$$

The experimental value for the dipole polarizability is $\alpha_{d}=$ 27.61 [28], and the cut off parameter $r c=1.63$ [27].
The total energy E of the system may be written, in Rydberg, as

$$
E=E_{T}+k^{2},
$$

where $E_{T}$ and $k^{2}$ are the energy of the target and the kinetic energy of the incident positrons, respectively.

$V_{\text {int }}(r, x)$ is the interaction potential between the incident positron and the target and is given by

$$
V_{\text {int }}(r, x)=\frac{2 Z}{x}-\sum_{i}^{N} \frac{2}{\left|x-r_{i}\right|}
$$

\section{RESULTS AND DISCUSSIONS}

The computation of the annihilation parameters was started by the calculation of the orbital wavefunctions and energies of the target atoms using Cowan computer code (program RCN32 ). These wavefunctions are used for the calculation of the positron-Xe atom interacting potential, then the matrix elements are calculated numerically.

The construction of the matrix elements $(S \mid S),(S \mid C)$, $\left(S \mid \phi_{i}\right),(C \mid S),(C \mid C),\left(C \mid \phi_{i}\right),\left(\phi_{j} \mid S\right),\left(\phi_{j} \mid C\right)$, and $\left(\phi_{j} \mid \phi_{i}\right)$ are used in the LSVM program (LLSQ) with starting value of the free parameter (non linear parameter) $\alpha$ and certain value of $\mathrm{n}$ (the dimension of the Hilbert-space part of the trial wavefunction). The optimum value of $\alpha$ is obtained from the plateau curve (the stationary behavior for R11 with $\alpha)$. The minimum value of $(H-E)\left|\Psi_{t}^{n}\right\rangle$ is found at $\alpha=$ 1.45 and $\mathrm{n}=13$. The s-wave elastic scattering cross-section $\sigma_{e l}\left(\pi a_{0}^{2}\right)$ of positron by xenon atom below the positronuim Ps formation threshold is drawn in figure (1) and compared with the results of semiempirical model calculated by Mitroy and Ivonove[2], and polarized orbital method calculated by McEachran et al [14]. For xenon, the Ps formation threshold is at $5.33 \mathrm{eV}$ (momentum $\sim 0.63 a_{o}^{-} 1$ ). The s-wave elastic scattering cross section (in $\pi a_{0}^{2}$ units) is related to the phase shift by the following relation

$$
\sigma_{e l}=\frac{4}{k^{2}} \operatorname{Sin}^{2}\left(\eta_{0}\right)
$$

The figure shows the elastic scattering $\sigma_{e l}$ decreases rapidly as the energy of the incident positron increases from zero to Positronuim threshold.

The electrons and positron wavefunctions are used to compute the annihilation parameter $Z_{e f f}$ for valence and inner subshells. The total $Z_{\text {eff }}$ are found by adding the contribution of different subshells using equation (3) which can be rewritten as:

$$
\begin{aligned}
& Z_{e f f}(k)=\frac{1}{4 \pi} \int d r\left[\operatorname{Sin} k r+R_{11} \operatorname{Cos} k r\left(1-e^{-\alpha r}\right)\right. \\
& \left.+\sum_{i=1}^{n} d_{i} e^{-\alpha r} r^{i}\right]^{2} \sum_{j}\left(R_{n_{j} \ell_{j}}\right)^{2}
\end{aligned}
$$

The annihilation parameter $Z_{e f f}$ is computed numerically .It is found that it is sensitive to the chosen wave function parameters. 


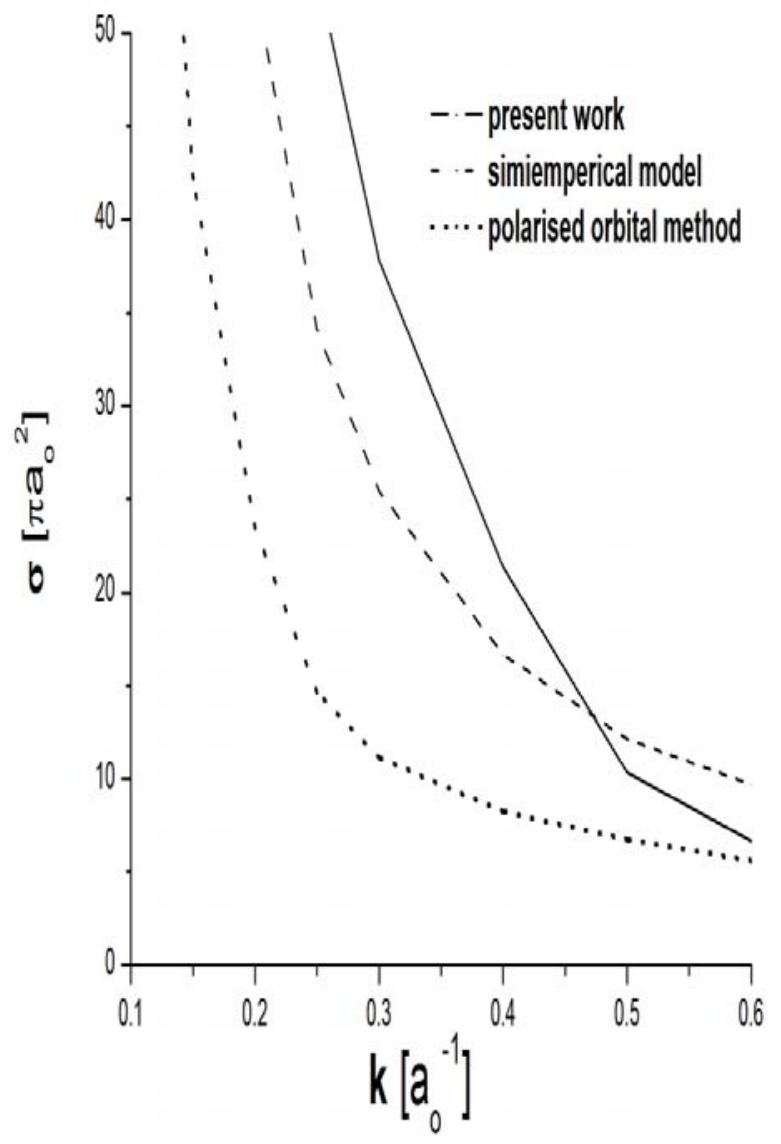

FIG. 1: The s-wave cross section for positron-xenonn scattering as a function of positron momentum $\mathrm{k}$ in (a-1). The solid line are the present results. The dashed lines are the results of semiempirical model calculated by Mitroy and Ivonove [2]. The dotted lines are the results of polarized orbital method calculated by McEachran et al [14]

At low energies the dominant contribution to annihilation parameter $Z_{\text {eff }}$ is from s-wave scattering. The dependence of the total $Z_{\text {eff }}$ on the positron momentum, $k$, over the range $0=k=1.2$ which are plotted, together with the total $Z_{e f f}$, in figure (2). This figure shows that our theoretical calculations have the same behavior as that calculated by semi-empirical model [2], polarized orbital method [14] and the experimental work of J.P. Marler et al[16]. The annihilation parameter Zeff reveal an initial decrease as positron energy is increased from zero, instead of $Z_{e f f}$ continuing to decrease, it starts to increase at positron energies just below the positronuim formation threshold. The annihilation parameters $Z_{\text {eff }}$ is about 150 for positron temperature $293 \mathrm{~K}$ (thermal energy $\sim 0.024$ $\mathrm{eV}$, momentum $\sim 0.042 a_{o}^{-1}$ ) which means that at room temperature probability that the positron annihilate is great. The present value of Zeff is less than the measurements of UCL group [29,30] which is 400-450 and the measurements of San Diego group, $\left(Z_{\text {eff }}=400\right)$ [31,32].

For positron momentum greater than $\sim 0.4 a_{o}^{-1}$, the curve falls, this needs explanation. More improving of the wave function through increasing the number of terms and taking the virtual Positronuim formation into account can help. When positrons interact with atoms they annihilate predom-

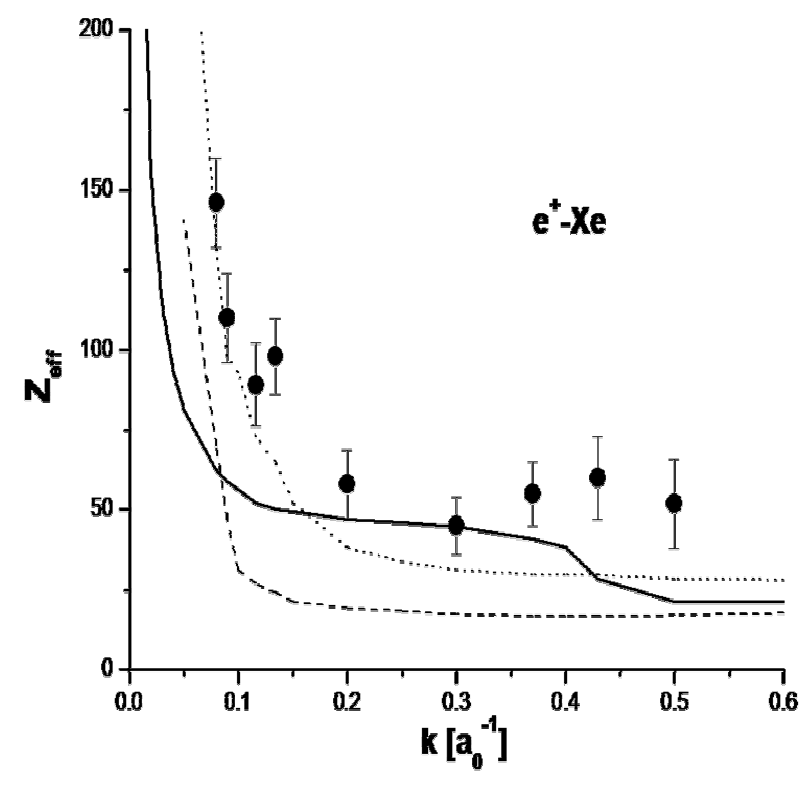

FIG. 2: The momentum dependence of Zeff of positron. The solid line are the present results. The dotted lines are the results calculated by Mitroy and Ivonove[2]. The dashed lines are the McEachran et al results [14]. The solid circles are the experimental work of Marler et al [16].

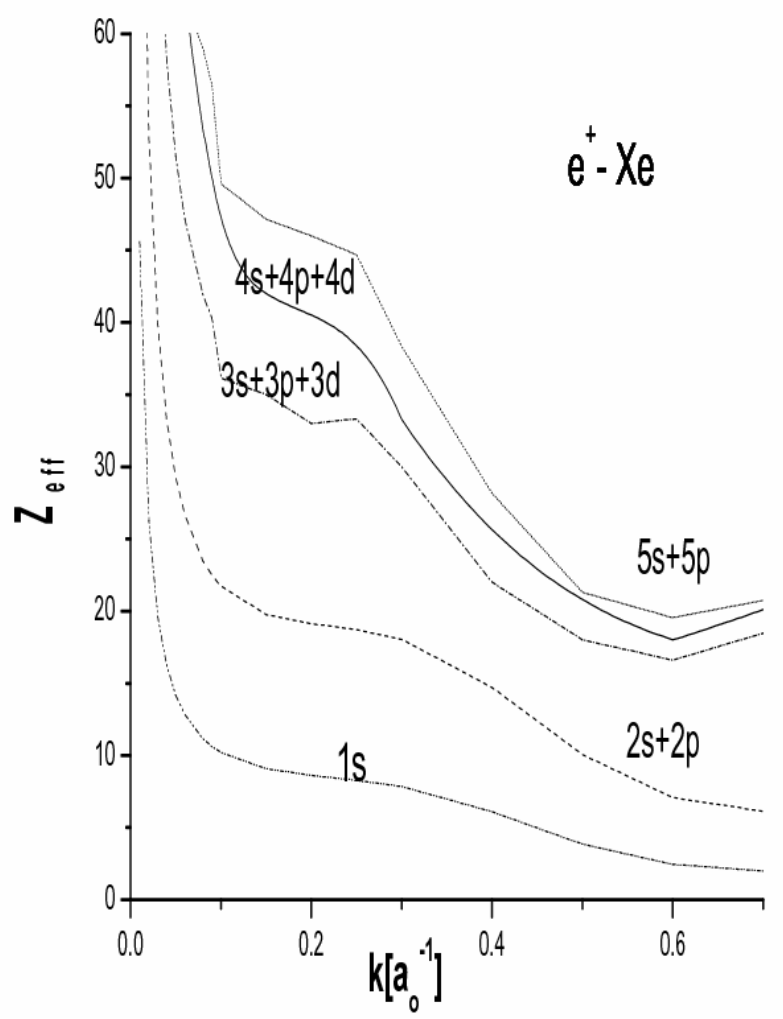

FIG. 3: The momentum dependence of the annihilation parameter Zeff of positron with different shells of positron - Xe atoms 
inantly with valence electrons, since the repulsive force between the positrons and nucleus keeps the positrons away from inner electrons. However small fraction of positrons can tunnel to inner electrons region and annihilate with some of them. The annihilation parameter $Z_{\text {eff }}$ have calculated at each shell of the Xe-atom using equation (28). The contribution of them to the annihilation parameter $Z_{e f f}$ are shown in figure (3).

Figure (3) shows that positrons annihilate predominantly with electrons of upper shells. For the principal quantum numbers $n=3$ and $n=4$ the annihilation parameter $Z_{\text {eff }}$ is roughly proportional to the number of electrons in the $s$, $p$, and $d$ subshells, namely, 2, 6, and 10 , respectively. The $Z_{\text {eff }}$ of $1 \mathrm{~S}$ shell is expected to become practically zero, as the positron momentum approaches zero since the positron would have no kinetic energy to penetrate the inner shells region. From figures the $Z_{\text {eff }}$ of $1 \mathrm{~S}$ shell is the same behavior as the experimental and theoretical $Z_{\text {eff }}$ which is great at zero positron energy and then decreases rapidly as the positron energy increase to Positronuim threshold. This paradox needs more theoretical and experimental work to solve. The lifetime

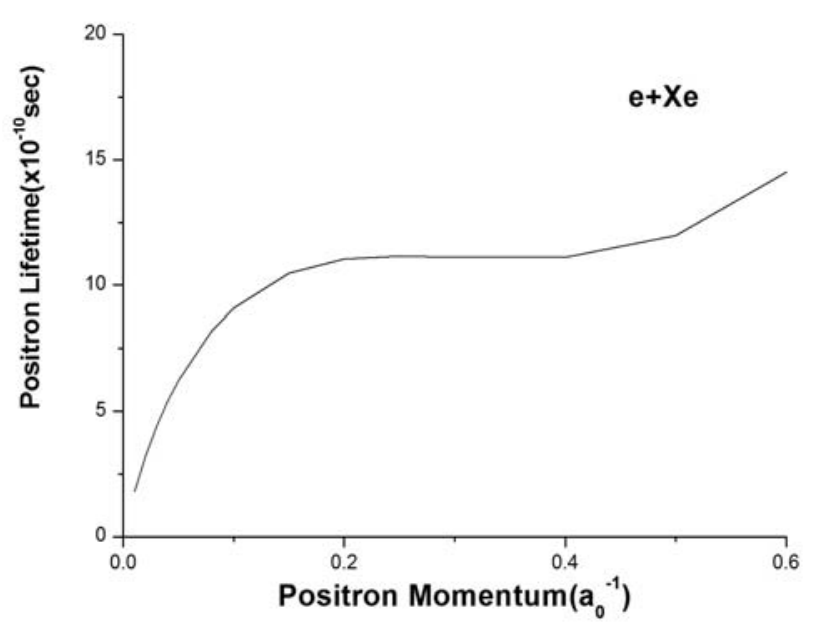

FIG. 4: The dependance of positron lifetime on its momentum of free positron in xenon atom is drawn in figure (4). The figure demonstrates the monotonic increase of the lifetime as the momentum of the incident positron increases, but it starts to decrease slowly as the positron momentum approach the Positronuim formation threshold.

\section{CONCLUSION}

In this work I have shown how the least-squares variational method (LSVM) can be developed for scattering wave function which used to calculate the annihilation rate of positrons in xenon atom as well as the lifetime. Hartree-Fock-Slater method is used to generate the orbital wavefunctions for xenon atom The minimum value of $(H-E)\left|\Psi_{t}^{n}\right\rangle$ is found at $\alpha=1.45$ and $n=13$. The electron and positron wavefunctions are used to compute the annihilation $Z_{e f f}$. The contributions of $Z_{e f f} Z_{e f f}$ has calculated numerically for valence and inner subshells of Xe-atom. It is found an agreement with a previously calculated and experimental values. The leastsquares variational method (LSVM) is applied successfully for positron-Xe in this work. The least-squares variational method (LSVM ) can be applied safely to positron-atom scattering.
[1] C. M. Surko, G. F. Gribakin, and J. Buckman, J. Phys. B 38, R57 (2005).

[2] J. Mitroy and I. A. Ivonove, Phys. Rev. A65, 042705 (2002).

[3] D. M. Schrader and Y. C. Jean, editors, Positron and Positronuim Chemistry, Amsterdam, Elsevier(1988).

[4] M. Dimanchev, A. Shofan, T. Troev and J. Serna, Material Science Forum 105 (1992).

[5] Y. C. Jean, and Q. Deng J. Polym Sci. B30, 1359 (1992).

[6] C. S. Sunder, A. Bharathi, W. Y. Ching and Y. C. Jean, Phys. Rev. B 42, 2193 (1990).

[7] K. O. Jensen and A. Weiss, Phys. Rev. B41, 3928 (1990).

[8] J. Liuand and Y. C. Jean, American Chemical Society 28, 5774 (1995).

[9] Z. Tang et al., Phys. Rev. Lett. 78, 2236 (1997).

[10] J. P. Peng K.G. Lynn, P. Asoka-Kumar, D.P. Becker and D.R. Harshman, Phys. Rev. Lett. 76, 2157 (1996).

[11] A. Weiss, R. Mayer, M. Jibaly, Y.C. Lel, D. Mehl, K. G. Lynn, Phys. Rev. Lett. 61, 2245 (1988).
[12] G. Sinapius, W. Raith and W. G. Wilson, J. Phys. B: At. Mol. Phys. 13, 4079 (1980)

[13] V. A. Dzuba, V. V. Flambaum, G. F. Gribakin and W. A. King, J. Phys. B: At. Mol. Opt. Phys. 293151 (1996)

[14] R. P. McEachran , A. D. Stauffer and L. E. M.Campbell, J. Phys. B: At. Mol. Phys. 13, 1281 (1980).

[15] T. J. Murphy and C. M. Surko J. Phys. B: At. Mol. Opt. Phys. 23 727(1990)

[16] J.P. Marler, L.D. Barnes , S.J. Gilbert , J.P. Sullivan , J.A. Young, C.M. Surko, Nucl. Instrum. Meth. Phys. Res. B 221, 84 (2004)

[17] M.A. Abdel-Raouf, Phys.Rep.108 pp1-164(1984).

[18] M.M. Abdel-Mageed, M.A., Abdel-Raouf, and S.Y. El-Bakry, Egypt. J. Phys. 38, 153-167 (2007)

[19] M. M. Abdel-Mageed, M. Abdel-Aziz, and H.S. Zaghloul, AIP Conference Proceedings. 888, 358 (2007).

[20] Ali H. Moussa, G. M. Mostafa and Atef Khazbak, J. Quant. Spect. and Rad. Tran., 77, 225 (2003). 
[21] M.A.El-Aasar, M.A. and Abdel-Raouf, J. Phys. B: At. Mol. Phys 40, 1801 (2007).

[22] J. W. Humberston Adv. At. Mol. Phys. 15, 101 (1979).

[23] J. W. Humberston and J. B. G. Wallace, J. Phys. B: At. Mol. Phys. 51138 (1972).

[24] Robert D.Cowan, "The Theory of Atomic Structure and Spectra”, Univ. of California Press (1981); Phys. Rev.163, 54 (1967).

[25] E.W. Schmid and K.H. Hoffmann, Nucl. Phys. A 175(1971).

[26] E .W. Schmid, Nucl. Phys. A180, 434 (1972) ; Nuovo Cim. A18, 171 (1973).

[27] J. Mitroy and M. W. J. Bromley, Phys. Rev. A , 67, 034502
(2003).

[28] A. Kumar and W. J. Meath, Can. J. Chem.,36, 1616 (1985).

[29] P.G. Coleman, T. C,Griffih, G.R. Heyland, and T. L kileeen, J. Phys. B 8, 1734 (1975).

[30] G. L. Wright, M. Charlton, T. C. Griffih and G.R. Heyland, J. Phys. B 18, 4327 (1985).

[31] T. J. Murphy, M. D. Tinkle and C. M. Surko, Phys. Rev. A 51, 473 (1995).

[32] I. Iwata, R. G. Gieaves, T. J. Murphy, M. D. Tinkle and C.M. Surko, Phys. Rev. A 51, 473 (1995). 